\title{
COMPARATIVE STUDY ON METHODS FOR 3D MODELLING OF URBAN AREAS
}

\author{
E. Oniga ${ }^{a}$ \\ ${ }^{\text {a }}$ Department of Terrestrial Measurements and Cadastre, Technical University „Gheorghe Asachi” of Iasi, D. Mangeron \\ Street, Iasi, Romania - ersylya83@yahoo.com
}

KEYWORDS: 3D model, Building, Laser scanning, Terrestrial Photogrammetry, Aerial Photogrammetry

\begin{abstract}
This article presents (comparatively) the methodology for creating a 3D model of the urban area, based on terrestrial laser scanner, traditional technologies of terrestrial photogrammetry and aerial images. We are reviewing the data sources, their preliminary processing to be brought in a common system and the software used for this purpose. The case study presents the comparative results obtained using the methods listed above. To obtain the 3D models with terrestrial laser scanner systems have been used the dates achieved with ScanStation 2. To obtain the 3D model drawn by traditional photogrammetric methods using the UMK terrestrial camera, have been used auxiliary dates from topographic measurements with GPS systems and total stations as well as the current topographic plans. For the 3D model creation based on the dates taken with aerial digital cameras, were used the aerial images, taken with the ADS40 photogrammetric aerial camera. The comparative study between the three methods was accomplished by analyzing the object space representation fidelity, the precision of the 3D models obtained by comparison of the distances, areas and volumes, comparing the execution time and execution costs. The article presents the conclusions, the advantages and disadvantages of the three technologies based on the criteria listed above.
\end{abstract}

\section{INTRODUCTION}

3D Modeling using multiple images represents one of the most atractive and provocative researching domain in photogrammetry and remote sensing. One practical application of the 3D modelling techniques is automatic generation of the digital model of the reflectance surface (MDSR), which, after being filtered produces the altimetric model of the terrain (MDA), of the digital surface of the terrain (Digital Surface Models (DSM)), using satelite, aerial or terrestrial images. Another important application that represents the object of this study is 3D modelling of the urban area.

In order to address our theme, we have created a 3D data base for the study area. The base level of these data consists in the precise topographic elevation captured with GPS systems and Leica TC(R) 405 total station.

This station is a high-quality electronic total station, ideally suited for simple construction surveys and setting out tasks. It allows the measurements without reflector, with the integrated visible laser beam, with a range of up to $100 \mathrm{~m}$. The accuracy in the angle measurement is 5 " and in the case of distances is $5 \mathrm{~mm} \pm 2 \mathrm{ppm}$.

The terrestrial photogrammetric measurements taken with UMK $10 / 1318$ camera have been inserted in this data base.

The $U M K 10 / 1318$ is a metric terrestrial camera and was built in 1973 by the Carl Zeiss Jena company. As a photosensitive material this camera uses photo plates $13 \times 18$, which have the useful format of the raw image $120 \mathrm{~mm} \times 166 \mathrm{~mm}$. The focal length of the camera is $100 \mathrm{~mm}$ with a focus setting from $\infty$ to 1.4 $\mathrm{m}$ for long-range photogrammetry (Linder, 2006). After the exposure one could notice that the raw images have a radiometric and geometric high quality.
For modern digital image processing methods to be applied, the raw images have been scanned. By adequately processing, based on the control points determined on the buildings surface the corresponding orthophotos are generated.

Separately, field works using ScanStation 2 have been made for the building in the testing area, for the purpose of 3D buiding model generation, based on this measurements.

The ScanStation2, produced by Leica Geosystems, is a terrestrial scanner system using 3D laser technology, used mainly in static measurement, being a scanning flexible solution which rapidly captures and measures all the details in a scene, not just what may be considered important at the time, ideal for complex environments (Leica brochure).

The specific processing of these observations from the field, using the data base previously created, permitted 3D model generation of the construction under discussion. For this 3D model the texture captured from non-metric digital photos of the facades has been added as a supplementary information.

The aerial images have been captured with ADS40 photogrammetric camera and we have obtained three band images in panchromatic domain, with the spatial resolution of $15 \mathrm{~cm}$ and four multi-spectral band images.

The ADS40 digital photogrammetric aerial camera, with a pushbroom sensor, offers enough informatio and a detailled description of the complex urban environment and they also raise the eficiency of the photogrammetric process. These images have been processed with ERADS - PRO 600, v11.0 system, obtaining the data for the 3D model by stereoscopic measurements. 
PRO600 is most commonly used for precise measurement, when engineering-level accuracy is required. It is nearly exclusively used for work with airborne imagery and is typically used with large-scale imagery. The editable feature definition library gives users full control over defining the types of features they are mapping and their characteristics (Erdas brochure).

All three 3D models obtained were exported to a CAD system such as AutoCAD software or MicroStation for the final processing.

At this stage the comparative appreciation has been made, regarding the levels of precision of the linear elements, surface, volume and also regarding the fidelity and complete reprezentation of the details of the buildings in the testing area.

\section{PRESENTATION OF THE STUDY AREA, MATERIALS, EQUIPMENT AND BASIC WORK}

The test zone is the „Department of Terrestrial Measurements and Cadastre" building, from the Technical University „Gheorghe Asachi" of Iasi, that has two building parts with different heights, both with a regular shape that is rectangular parallelepiped (the roof is flat).

\subsection{Materials and equipment}

The main data for this task are:

- the building ground plan;

- raw images taken with UMK 10/1318, scanned with the Epson V750 desktop scanner and processed with Lisa software;

- the "point cloud" achived with ScanStation 2 and processed with the Cyclone v. 6.0 software;

- digital aerial images taken with ADS40 and processed with ERDAS - PRO 600, v11.0 software.

- digital photos used for the texture generation of the building facades.

The utilized equipment is:

- South S82-V GNSS Rover,

- the TC(R) 405 total station;

- the ADS40 digital photogrammetric aerial camera and ground measurements with GPS systems,

- the digital photogrammetric equipment used in this article is the Digital Photogrammetry Workstation (DPW) equipped with ERDSAS - PRO 600, v11.0 software,

- the Cannon EF $50 \mathrm{~mm}$ lens, a 10 mega pixel camera with $10 \times$ optical zoom.

\subsection{Basic work}

The measurements for the traverse network points co-ordinates determination were carried out with the Leica TC(R) 405 total station and GPS systems. The network was defined by six stations, set permanent for futher use in the measurement of the control points. For obtaining the final stations co-ordinates the least square ajustment was used. After the measurements the study zone ground plan was obtained, needed for the realisation of the terrestrial raw images acquisition project (the ground mark of the base lines raw images acquisition) (Figure 1).

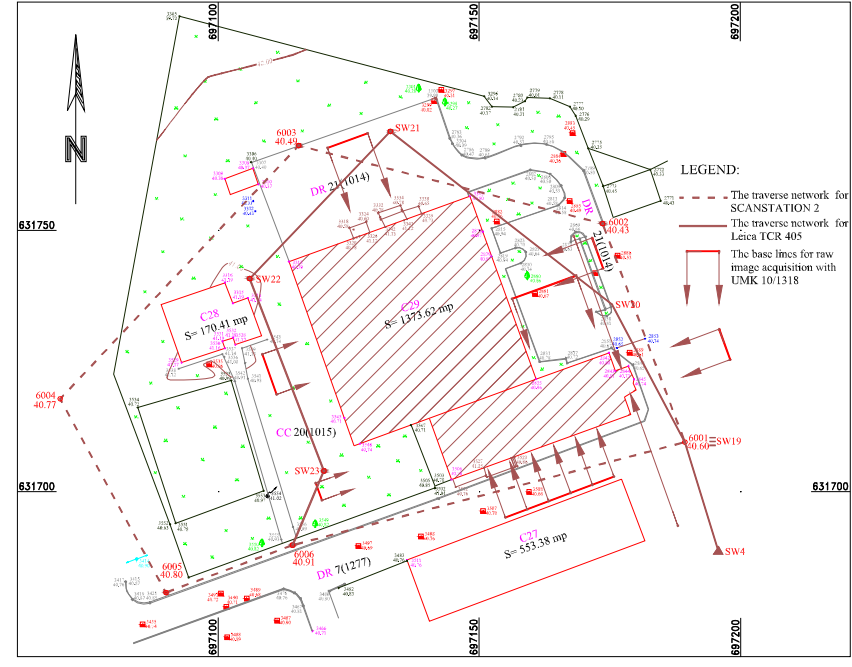

Figure 1. Building ground plan, the traverse scheme and the base lines for raw image acquisition

From the network points, the stereoscopic base lines points were ground marked (twenty camera positions) and more than 60 artificial control points (targeted points) placed on the building facade were measured with Leica TC(R) 405 total station reflector less measurement. An example of natural and artificial points is shown in Figure 2.

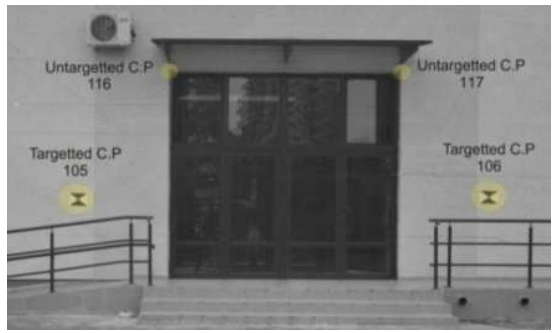

Figure 2. The location of some natural and artificial control points (North facade)

The measurement processing made for the control points, were performed with the TopoSys software, resulting the $(\mathrm{X}, \mathrm{Y}, \mathrm{Z})$ coordinates, with a precision of millimeter in all three coordinates, in National Projection System "Stereographical secant plan-1970" and the „Black Sea 1975” reference system for heights.

The co-ordinates of some control points, natural and artificial, located on the north facade are presented in Table 1.

\begin{tabular}{|c|c|c|c|c|}
\hline Point & $\mathrm{X}[\mathrm{m}]$ & $\mathrm{Y}[\mathrm{m}]$ & $\mathrm{Z}[\mathrm{m}]$ & Report \\
\hline 100 & 631756.130 & 697147.067 & 48.718 & Target Pt. \\
\hline 105 & 631752.422 & 697136.991 & 42.422 & Target Pt. \\
\hline 106 & 631750.142 & 697130.645 & 42.505 & Target Pt. \\
\hline 107 & 631744.339 & 697114.525 & 41.404 & Target Pt. \\
\hline 116 & 631751.934 & 697135.599 & 44.559 & Untargeted \\
\hline 117 & 631750.643 & 697132.014 & 44.575 & Untargeted \\
\hline
\end{tabular}

Table 1. The co-ordinates of control points 


\section{THE 3D MODEL GENERATION BASED ON TERRESTRIAL PHOTOGRAMMETRY}

\subsection{Photogrammetric Surveying}

With the help of the UMK 10/1318 photogrammetric camera about 25 images were taken in normal position (Figure 3 ), and additional approximate 100 digital photos with the Cannon EF 50 $\mathrm{mm}$ lenses digital camera.

Analogue UMK photographs (negative film) were scanned with a desktop scanner Epson V750 at 760.93dpi.

The total photographic operation was completed in approximately fourteen hours, including setting up the artificial control points, the measurement of the co-ordinates of these points and photography processing, considering that the terrestrial images acquisition and the control points measurement were done simultaneously, having a team of four people.
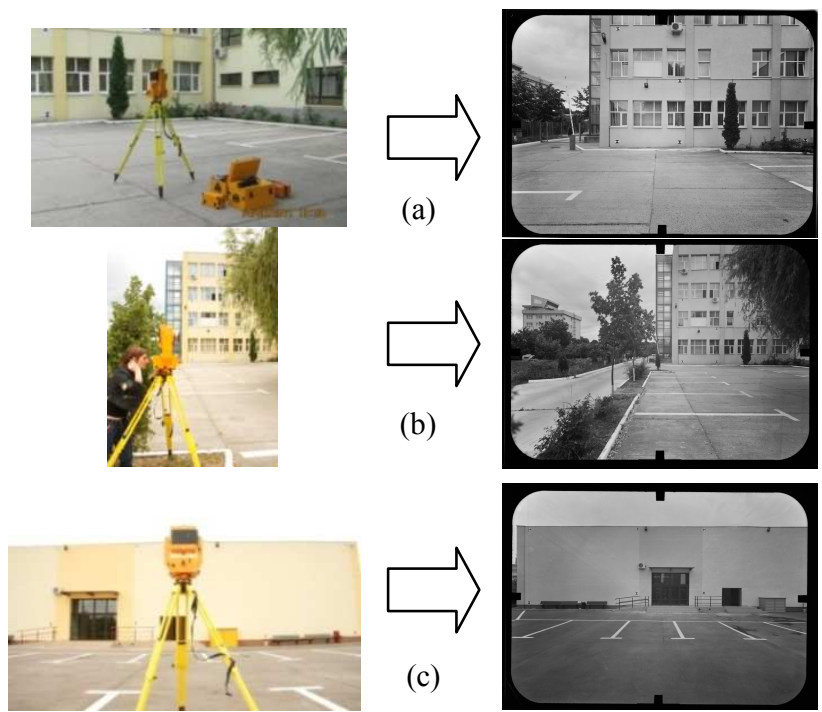

Figure 3. Data acquisition with the UMK 10/1318

photogrammetric camera (a), (b) North-East facade, (c) North facade

\subsection{Data from UMK 10/1318 Processing}

3.2.1. Orientation of the images: Because we have images coming from a film camera that were scanned. The first step to orient an image is the so-called interior orientation, which means establishing the relation between the camera-internal co-ordinate system and the pixel co-ordinate system. The first relation is given by the fiducial marks superimposed in the image and their nominal co-ordinates, usually given in $[\mathrm{mm}]$ in the camera calibration certificate (Linder, 2006). The images were scanned with 760.93 dpi resolution, which is equivalent with a value of $23.4 \mu \mathrm{m}$ of pixel size.

After we introduced the nominal co-ordinates of the fiducial marks and the focal length $(100,000 \mathrm{~mm})$, we digitized the marks and the software calculated the transformation coefficients (plane affine transformation) for the relation between both systems.
After we measured those four fiducial points (example: for north facade), were obtained the residual errors shown in Figure 4, which have the same values for $x$ and $y$ because only four points give a weak determination for the affine transformation (at least three points are necessary).

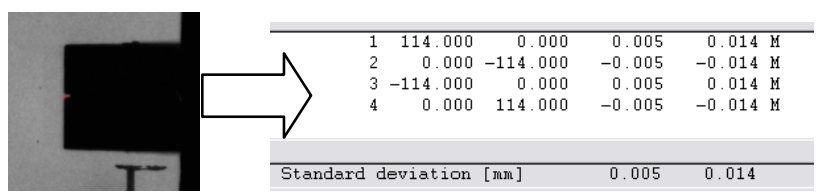

Figure 4. Remaining errors after the adjustment (north facade)

For each image the exterior orientation was carried out independently, interactively by measuring the control points. Residual errors were obtained for $x$ and $y$ for every point and also the standard deviation of the image, in [mm] (Figure 5).

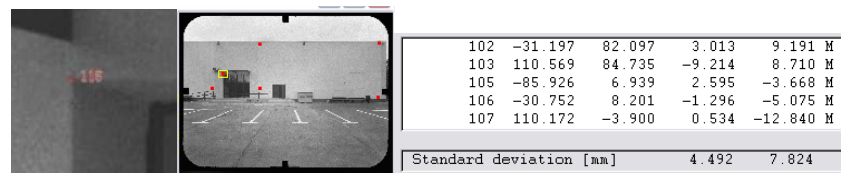

Figure 5. Remaining errors and the standard deviation after the exterior orientation (North facade)

3.2.2 Model definition: It is our goal to measure threedimensional object co-ordinates, so we created the model or the stereo pair for every two neighboring images. This means that if we have three-dimensional object (terrain) co-ordinates of a point inside the model area it is possible to calculate the pixel coordinates of this point in the left and the right image using the well-known collinearity equations (Linder, 2006):

$$
\begin{aligned}
& x^{\prime}=f \cdot \frac{r_{11}\left(X-X_{0}\right)+r_{21}\left(Y-Y_{0}\right)+r_{31}\left(Z-Z_{0}\right)}{r_{13}\left(X-X_{0}\right)+r_{23}\left(Y-Y_{0}\right)+r_{33}\left(Z-Z_{0}\right)} \\
& y^{\prime}=f \cdot \frac{r_{12}\left(X-X_{0}\right)+r_{22}\left(Y-Y_{0}\right)+r_{32}\left(Z-Z_{0}\right)}{r_{13}\left(X-X_{0}\right)+r_{23}\left(Y-Y_{0}\right)+r_{33}\left(Z-Z_{0}\right)}
\end{aligned}
$$

where: $(\mathrm{X}, \mathrm{Y}, \mathrm{Z})-$ the object point coordinates $\left(\mathrm{X}_{0}, \mathrm{Y}_{0}, \mathrm{Z}_{0}\right)$ - the projection coordinates center $\mathrm{r}_{11}, \mathrm{r}_{12}, \mathrm{r}_{21}, \mathrm{r}_{22}, \ldots$ - the coefficients of the rotation matrix $\mathrm{f}$ - the focal length

After the program correlate both images in well-known positions taken from the control point file, we are able to view the model stereoscopically, to measure the three-dimensional object coordinates and to digitize objects like points, lines or areas, sometimes called feature collection (Figure 6).

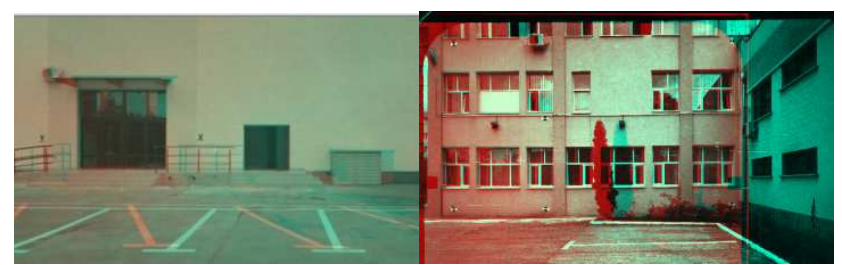

(a)

(b)

Figure 6 - Stereoscopic viewing (a) North facade, (b) North East facade 
To create the 3D model of the building, the digitization of its characteristic points was chosen (corners of windows, doors, etc.). As the output data of the program, was obtained a *.txt file which contains the points numbers and their $3 \mathrm{D}$ co-ordinates. The points were numbered in the measuring order, and the starting point was introduced by the operator. The *.txt file was then imported into the AutoCAD 2010 software, where the 3D model of the building has been created as following: point-line-surface-solid (Figure 7). For a better and a realistic view, the building facades texture were created using fragments from photo images. The 3D model of the building containing textures (south facade) is shown in Figure 8.

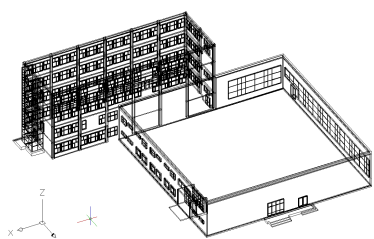

Figure 7. The 3D building model (view mode: wireframe)

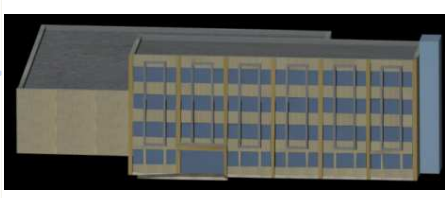

Figure 8 . The textured 3D model of the building (realistic view mode)
For the textured 3D model of the building generation, were utilized the digital photos taken with the Cannon EF $50 \mathrm{~mm}$ lens digital camera.

\subsection{Orthophoto Generation}

The first task, where photogrammetry is applied, is to make orthophotos of given facades. Orthophotos can be obtained by a orthorectification of images (Sadjadi, 2008). For the facades of this building, image orthorectification was found to be suitable, since all the facades could be projected onto a surface without accuracy loss. The analytical rectification has been made by means of the well-known two-dimensional projective transformation (Novack, 1992).

$$
\begin{aligned}
& u=\frac{a_{1} x \times a_{2} y+a_{3}}{c_{1} x \times c_{2} y+1} \\
& v=\frac{b_{1} x \times b_{2} y+b_{3}}{c_{1} x \times c_{2} y+1}
\end{aligned}
$$

where: $\quad \mathrm{u}, \mathrm{v}$ - image coordinates

$\mathrm{x}, \mathrm{y}$ - planimetric object coordinates

$a_{1}, a_{2}, a_{3}, b_{1}, b_{2}, b_{3}, c_{1}, c_{2}-$ projective transformation coefficients

Resampling was made by bilinear transformation.

\subsection{Mosaicking}

Most facades could not be taken from a single image, so it was necessary to make the mosaics from rectified images. To make the mosaic the control points both natural and artificial were used, which were necessary for the projective transformation coefficient calculation. All the photos must be edge matched, both visually and by coordinates, no edge match tolerance may be allowed. Finally, a radiometric adjustment was made to homogenize the mosaics.

In figures 9 and 10 the mosaics corresponding to the north-east and, respectively, the north facades of the building are presented.
To achieve the mosaics 6 images were used, taken with the UMK $10 / 1318$ terrestrial photogrammetric camera.

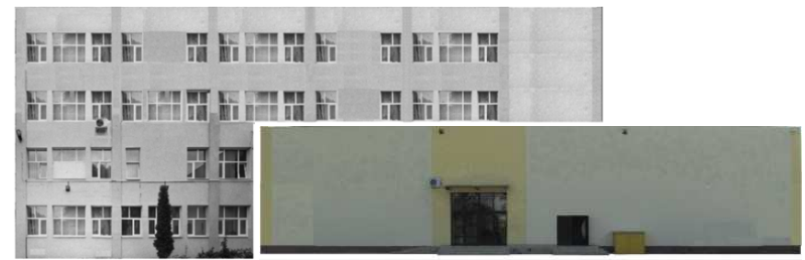

Figure 9. Rectified mosaic image of the north-east facade (from UMK raw images)

Figure 10. Rectified image of the north facade (from Cannon images)

Digital terrestrial photogrammetry is considered a method allowing many close-range measurement applications that permits rapid data recording at low-cost relative to other measurement techniques, flexibility and high measurement accuracies (Sadjadi, 2008).

\section{THE 3D MODEL GENERATION BASED ON TERRESTRIAL LASER SCANNER}

\subsection{Terrestrial Laser Scanner Surveying}

For this case study, the „Department of Terrestrial Measurements and Cadastre" building was scanned in three hours, achieving five scans (ScanWorlds) taken from five different scanning stations (Figure 1), each of them lasting approximately 30 minutes. The scanning stations were made between SW19 and SW23 support points, as a tachometric traverse, having the bases (SW19-SW4), respectively (SW23-6006), with known orientation. From SW19, SW20, SW21, SW22 and SW23 points the scanning operation was made, and on the SW4 and 6006 the circular targets were centered (of Leica type). During scanning a field notebook was made. For bringing all the 5 stations in a common system the direct georeferencing process was necessary. During the scan process were introduced: the start points elements- the co-ordinates of the SW19, SW4 points, the instrument height and the target height, in each station point- the instrument height and the targets heights; the arrival points elements - the co-ordinates of the SW23, 6006 points, the instrument height and the target height. The data base obtained after the georeferencing process (Iasi.imp) was imported in the Cyclone software for data processing.

\subsection{Data from ScanStation2 Processing}

4.2.1 Noise filtering: An important operation in post-processing is the filtering of the resulted data, which presumes the elimination of the points which are not part of scanned, deleting points which contain noises generated by the wind, the weak reflection from the scanned surface, obstacles of moving persons or objects, the scanning resolution and so on. In this way, the measured points, additionally taken by the instrument, were manually eliminated, being easily identified by analyzing the scanned area. In figure 12 is shown the point cloud obtained after filtering which contains information regarding the reflectance values of laser beams and information about the real color under RGB values (Red, Green, Blue). 


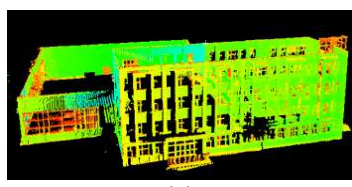

(a)

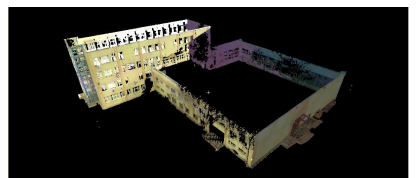

(b)
Figure 11. The point cloud for the study building after the filtering process containing information about (a) the reflectance (b) the real color

4.2.2 The point cloud modeling: Modeling the point cloud was performed by using lines and surfaces with the existing functions in the Cyclone v. 6.0 software. Modeling started with the segmentation procedure where the scanned points were approximately grouped according to the plan they belong. There were defined areas built by a closer point group which fit well to a plan and they define distinct elements of the facade. The surfaces created in Cyclone were exported as a *.dxf file which was imported into AutoCAD 2010 platform and after that the 3D model of the building was created and the distinct elements were organized on layers. To obtain a better representation, the textures were created using fragments from digital photos of the building facades (Figure $12 \mathrm{~b}$ ).

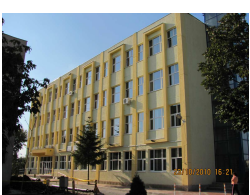

(a)

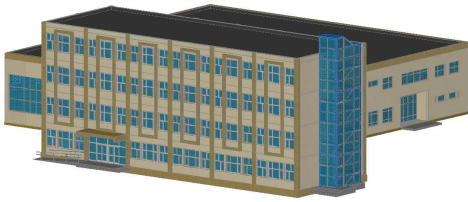

(b)
Figure 12. (a) Digital image of the building facade (b) The 3D model of the building after applying texture (South-East view)

\section{THE 3D MODEL GENERATION BASED ON AERIAL IMAGES}

\subsection{Data from ADS 40 Processing}

The digital data captured with ADS40 in may 2006 at the relative height of $1500 \mathrm{~m}$, cover all the Iasi city area. For photogrammetric exploatation, a digital aerial triangulation has been made using the panchromatic images. From the initial data we have extracted an strip image for the studied area. After the stereoscopic measurements, using the ERDAS PRO 600 v.11.0 sofware, the necessary data to create the $3 \mathrm{D}$ model of the building have been captured. The roof images from the aerial photograms were inserted into the new created 3D model (Figure $13 \mathrm{~b}$ ).

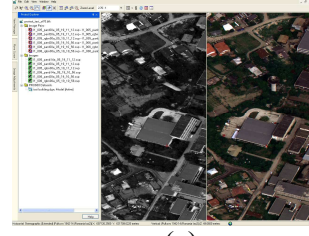

(a)

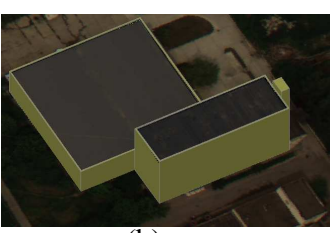

(b)
Figure 13. (a) PRO 600-ViewPlex window, (b) The 3D model of the building

\section{RESULTES AND DISCUTIONS}

The insertion of the obtained data in a data base facilitates the analisys, appreciation and editing of the results. Each one of the 3D models obtained as we have shown above can be improved and become more efficient regarding the precision of representation and viewing conditions. We have keep in mind the aspects related to the generalisation of the $3 \mathrm{D}$ model, the fidelity of the representation and the complete representation of the spaceobject, when they are compared.

In Table 2 are listed the facades surfaces of the building, obtained by using the three methods, and also the volumes of the building. We can observe that the maximum differences between the surfaces, obtained by using the first two methods do not come over $0.15 \%$ and between the last two methods, the maximum differences are $3.4 \%$. For the volumes, the difference between the first two methods is $0.003 \%$ and between the second and the thrid one the difference is $2.9 \%$. These differences are caused by the fact that the differences between the measured distances using the first two methods are $1 \div 2 \mathrm{~cm}$, and those between the measured distances by using the last two methods are $15 \div 30 \mathrm{~cm}$.

\begin{tabular}{|c|c|c|c|}
\hline \multirow{2}{*}{$\begin{array}{c}\text { Surface } \\
\text { No. }\end{array}$} & \multicolumn{3}{|c|}{ Surfaces $\left[\mathrm{m}^{2}\right] /$ Volumes [mc] } \\
\cline { 2 - 4 } & UMK 10/1318 & ScanStation 2 & ADS 40 \\
\hline 1 & 575.0581 & 574.9252 & 589.9428 \\
\hline 2 & 196.3733 & 196.5699 & 202.4070 \\
\hline 3 & 394.3292 & 394.8291 & 402.4933 \\
\hline 4 & 304.7870 & 304.3550 & 311.6010 \\
\hline 5 & 304.1975 & 303.9498 & 311.1531 \\
\hline 6 & 308.8039 & 308.6162 & 315.9714 \\
\hline 7 & 125.7151 & 125.8986 & 129.8615 \\
\hline 8 & 197.1843 & 196.9347 & 203.5854 \\
\hline- & 18537.3710 & 18536.8550 & 19062.6996 \\
\hline
\end{tabular}

Table 2. The surfaces and the volumes calculated through the methods listed above

\section{CONCLUSIONS}

This article shows how to create the 3D building model, through terrestrial laser scanner technology using the ScanStation2, traditional photogrammetric methods using the UMK10/1318 terrestrial photogrammetric camera and as well as through aerial digital images from ADS40, the methods used being complementary and being able to complete the reprezentation of the whole object-space.

\section{Advantages of traditional photogrammetric methods utilization Photogrammetry may be the best of available measuring} techniques from a narrow point station of view.

The digital data captured by terrestrial stereo photogrammetry can be used to create $3 \mathrm{D}$ wire-frame models that could be used as a basis for producing the $3 \mathrm{D}$ visualization of the buildings, the user 
being able to perform the whole reconstruction of the $3 \mathrm{D}$ buildings measurements.

\section{Disadvantages of traditional photogrammetric methods utilization}

The density of 3D measurements from photogrammetry is, which can be converted to a CAD model only after extensive interactive editing. In order to be processed by digital technologies, the method requires additional processing like scanning, thus inducing new sources of deformation which proved to be uneconomical and time consuming.

In contrast with laser scanning technology, the ground measurements are very laborious, lasting fourteen hours comparative with three hours in the laser scanner case.

Pitched roofs of the buildings can not easily be taken from ground stations.

\section{Advantages of laser scanner technology utilization}

Similar to photogrammetry, laser scanning is a noncontact measurement method and is increasingly used for creating the buildings or monuments 3D model.

For complex and irregular shaped objects terrestrial laser scanning is preferred. These objects can be scanned with high precision and we can obtain all the object details. Moreover, in this case, the results can be presented and viewed much better than the results of other photogrammetric methods.

The time for making measurements with scanning systems is considerably reduced, compared to terrestrial photogrammetry method.

The ScanStation2 system enables the acquisition of highresolution photos that can be referenced and used as support for data analysis and textures acquisition which are necessary in the modeling process.

\section{Disadvantages of laser scanner technology utilization}

The modeling part, or the process of transforming point clouds from the raw shape in complex models (with textures), it's done interactively with specialized software and it's time-consuming. The benefits of the laser scanning technology are reflected in the cost of the work, which is high.

\section{Advantages of aerial images technology utilization}

The aerial photogrammetry is extensively experienced and the stereoscopic evaluation is based on the mathematical model. The $3 \mathrm{D}$ model is drawn base on the 3D aerial images exploitation. The process can be automated to identify the corresponding points, lines or surfaces in the stereogram.

It requires a minimum of field work for the photograms evaluation (the control points densification for the evaluation being performed by aero triangulation).
Provides textural information regarding the roofs of the buildings.

The 3D model accuracy depends on the spatial resolution.

\section{Disadvantages of aerial images technology utilization}

The building facades should be inserted from terrestrial photogrammetry or laser scanner.

Field work is needed for the insertion in the 3D model of the elements that can not be clearly identified in the stereogram.

\section{REFERENCES}

References from Journals:

Mata, E., Cardenal, J., Castro, P., Hernandez, M.A., Perez, J.L., Ramos, M., Torres, M., -Digital and Analytical Photogrammetric Recording Applied to Cultural Heritage, a Case study: "St. Dominique de Silos' Church $\left(\mathrm{XIV}^{\text {th }}\right.$ Century, Alcala la Real, Spain)". The International Archives of the Photogrammetry, Remote Sensing and Spatial Information Sciences, Istanbul, Turkey,Vol. 34, Part XXX.

Sadjadi, S.Y., 2008 - An Integration of Close-Range Photogrammetry and Cad System for Cultural Monuments: Preliminary Findings, The WSEAS International Conference on engineering mechanics, structures, engineering geology (EMESEG ,08), Heraklion, Crete Island, Greece, July 22-24, 2008, pp. 142-150.

Novack, K., 1992 - Rectification of Digital Imagery, Photogrammetric Engineering and Remote Sensing, 58 (3), pp. 339-344.

References from Books:

Gutu, A., Andrei, O., 1976- Terrestrial photogrammetry in research and design, Technical Publishing Bucharest.

Linder, W., 2006 - Digital Photogrammetry, Spriger-Verlag, Berlin, Heidelberg.

McGlone, J., 2004. Manual of Photogrammetry. Fifth Edition, American Society for Photogrammetry and Remote Sensing.

Kasser, M., \& Egls, Y., 2002. Digital Photogrammetry. Taylor \& Francis, London.

\section{References from websites:}

www.erdas.com

www.leica-geosystems.com

\section{ACKNOWLEDGEMENTS}

I would like to express my sincere gratitude to prof. Florea Zavoianu for his unconditional support and contribution to the presented work. I am thankful to Dumitru Onose, Adrian Savu and Aurel Negrila (UTCB, Romania), for their help in scanning the building and to Bejenaru Matei, from University of Arts "George Enescu" Iasi, for his help in processing the UMK images. 